\title{
Third molars may have a negative impact on periodontal health
}

\author{
Is there an association between the presence of visible third molars and \\ periodontal pathology in a sample of middle-aged and older adults?
}

\begin{abstract}
Elter JR, Offenbacher S, White RP, Beck JD. Third molars associated with periodontal pathology in older Americans. J Oral Maxillofac Surg 2005; 63:179-184

Design This was a cross-sectional study.

Materials and methods Data were within a cohort study obtained between 1996 and 1999 on 6793 people of 52-74 years of age from the dental substudy of the Atherosclerosis Risk in Communities Study. The main independent variable was presence or absence of third molars, assessed visually, and the dependent variable was assessment of periodontal disease designated by pocket depth of $5 \mathrm{~mm}$ or greater $($ PD5 +). Associations were determined using odds ratios and $95 \%$ confidence intervals $(\mathrm{Cl})$. Weighted multivariable models were fitted using logistic regression and variances were adjusted to account for the clustering of quadrants within individual subjects.
\end{abstract}

Results A visible third molar was associated with 1.5 -fold $(95 \% \mathrm{Cl}$, 1.4-1.7) the odds of PD5 + on the adjacent second molar, while controlling for other factors associated with the presence of third molars and periodontal disease. Other factors positively associated with PD5 + in the model were male gender, older age, smoking, and irregular and episodic dental visits.

Conclusions The finding of more severe periodontal conditions associated with visible third molars in these middle-aged and older adults indicates that third molars may continue to have a negative impact on periodontal health well into later life. The relationship between third molars and periodontal disease pathogenesis deserves further study using longitudinal data.

\section{Commentary}

Over the years the humble wisdom tooth has come in for a fair amount of attention in the literature. This mass of dentine and enamel, essentially the same as any other tooth in the dentition, has been blamed for problems such as lower incisor crowding, atypical facial pain, caries, and even disorientation in aircrew! This paper presents data drawn retrospectively, and severe periodontal conditions are ascribed to the presence of visible third molars in middle-aged and older adults.

It is tempting to conclude that these patients had suffered from destructive periodontal disease and that this was likely to continue, suggesting that wisdom teeth might be better extracted — although this conflicts with the lack of evidence justifying their prophylactic removal. The parameters used here, however, were the presence or absence of at least a $5 \mathrm{~mm}$ probing depth and of gingival bleeding. The former may be a sign simply of "false pocketing" due to increased tissue thickness, commonly associated with partly- erupted third molar teeth. Indeed, it is common practice to exclude third-molar data from clinical trials because the probing depths found are often clinically meaningless. Similarly, the presence of gingival bleeding as a sign of gingivitis rather than periodontal destruction is well established. The most plausible explanation for this association might be that patients find it difficult to clean distal surfaces of second molars even after removal of the third molars and that this be attributed simply to their relative inaccessibility.

Previous work has indicated that malpositioning of teeth, as such, did not enhance periodontal breakdown but that it decreased the effect of average plaque control measures. Patients with exceptionally good oral hygiene or, on the other hand, no oral hygiene at all were less likely to reveal an association between tooth malpositioning and periodontal disease. ${ }^{1,2}$ No reference was made to plaque control in the current investigation, although the authors concede that plaque accumulation may have resulted in the gingival tissue changes found. They failed to demonstrate that these had resulted in periodontal attachment and bone loss. Varying degrees of third molar impaction could lead to altered hard and soft tissue anatomy without indicating active destruction. The authors conclude that "clinical indicators of periodontal disease" were more likely to be detected on adjacent third molars. Perhaps, more than anything, this study indicates the difficulty currently experienced by clinicians and researchers in ascribing a diagnosis of periodontitis.

\section{Practice points}

- The presence of visible third molars may be associated with apparent periodontal pathology which could simply be increased tissue thickness related to tooth position.

- Patients will usually need specific help with their plaque control in third molar areas.

- There is not yet, therefore, any evidence of benefit from the removal of third molars in order to prevent periodontal breakdown.

\section{Graham J Smart}

Postgraduate Dental Education Institute, Oxford, UK

1. Ainamo J. Relationship between malalignment of teeth and periodontal disease. Scand I Dent Res 1972; 80:104-110.

2. Buckley LA. The relationships between malocclusion, gingival inflammation, plaque and calculus. J Periodontol 1981; 52:35-40.

Evidence-Based Dentistry (2005) 6, 95.

doi:10.1038/sj.ebd.6400339 